\section{International Scientific Journal Theoretical \& Applied Science}

p-ISSN: 2308-4944 (print)

e-ISSN: 2409-0085 (online)

Year: 2016

Issue: 8

Volume: 40

Published: $30.08 .2016 \quad \underline{\text { http://T-Science.org }}$

SECTION 2. Applied mathematics. Mathematical modeling.
Alexandr Shevtsov candidate of technical sciences, member of PILA (USA), member of European Academy of Natural History (UK), corresponding member of the Kazakhstan National Academy of Natural Sciences, President of International Academy TAS, Department of «Mathematics», Deputy Director on Science of faculty of information technologies, automation and telecommunications, Taraz state University named after M.Kh. Dulati, Kazakhstan $\underline{\text { Shev_AlexXXXX@mail.ru }}$

\title{
ON SOME ALGORITHMS FOR PROGRAMMING ROBOTS
}

Abstract: The article considers the problem of robot programming with the possibility of gas-chemical analysis of air for dangerous concentrations of combustible gases.

Key words: robot, programming, gas.

Language: English

Citation: Shevtsov A (2016) ON SOME ALGORITHMS FOR PROGRAMMING ROBOTS. ISJ Theoretical \& Applied Science, 08 (40): 79-86.

Soi: http://s-o-i.org/1.1/TAS-08-40-16

Doi: crossef http://dx.doi.org/10.15863/TAS.2016.08.40.16

\section{Introduction}

As a sample we consider a wheeled robot "Observer - chemical reconnaissance".

\section{Brief description.}

Small robot upravlenii remotely videonabljudenie and chemical analyzers air for the presence of high concentrations of hazardous and flammable gases.

\section{Functionality:}

- covert surveillance of the area

- remote control

- chemical analysis of air for dangerous concentrations of gases (propane, methane, n-butane, smoke, hydrogen, natural gas, petroleum gas, butane, carbon dioxide, carbon monoxide, ammonia, benzene, nitrogen oxides, vapours of alcohol), temperature, humidity.

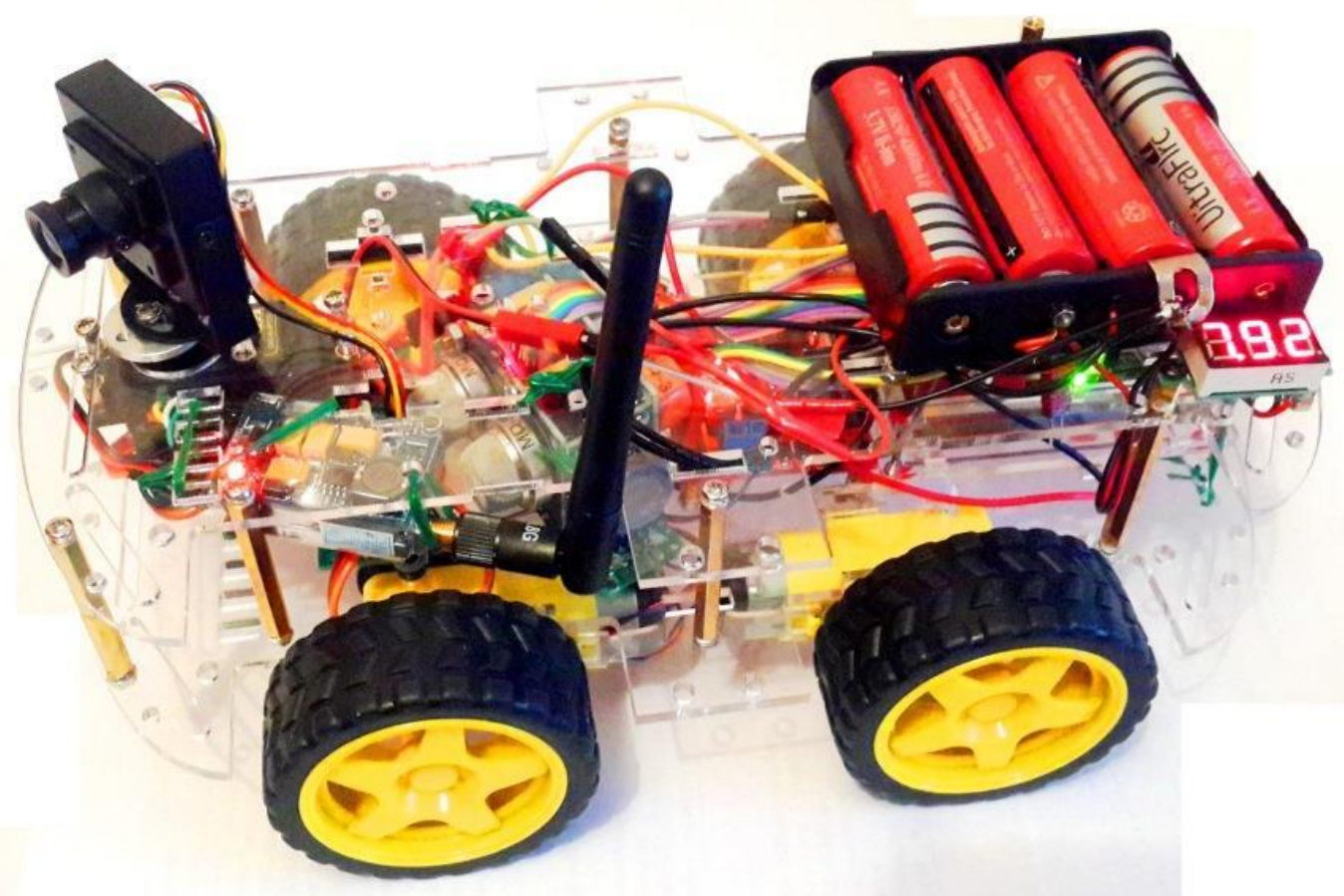

Figure 1 - Wheeled robot "Observer - chemical reconnaissance". 


\begin{tabular}{|c|c|c|c|c|c|c|}
\hline Impact Factor: & $\begin{array}{l}\text { ISRA (India) } \\
\text { ISI (Dubai, UAE } \\
\text { GIF (Australia) } \\
\text { JIF }\end{array}$ & $\begin{array}{l}=1.344 \\
=0.829 \\
=0.564 \\
=1.500\end{array}$ & $\begin{array}{l}\text { SIS (USA) } \\
\text { PИНЦ (Russia) } \\
\text { ESJI (KZ) } \\
\text { SJIF (Morocco) }\end{array}$ & $\begin{array}{l}=0.912 \\
=0.234 \\
=1.042 \\
=2.031\end{array}$ & $\begin{array}{l}\text { ICV (Poland) } \\
\text { PIF (India) } \\
\text { IBI (India) }\end{array}$ & $\begin{array}{l}=6.630 \\
=1.940 \\
=4.260\end{array}$ \\
\hline
\end{tabular}

Table 1

The performance characteristics of wheeled robot "Observer - chemical reconnaissance".

\begin{tabular}{|c|c|c|}
\hline № & option & value \\
\hline 1 & Dimensions (length, width, height) & $0,28 \mathrm{~m} / 0,17 \mathrm{~m} / 0,2 \mathrm{~m}$ \\
\hline 2 & Clearance & $0,015 \mathrm{~m}$ \\
\hline 3 & Weight & $1,2 \kappa \Gamma$ \\
\hline 4 & Maximum speed & $1.4 \mathrm{~m} / \mathrm{c}(5 \mathrm{~km} / \mathrm{h})$ \\
\hline 5 & The carrying capacity & $\sim 1 \mathrm{\kappa} \Gamma$ \\
\hline 6 & Remote control & $\begin{array}{l}\text { from computer, } \\
\text { with the tablet } \\
\text { smartphone }\end{array}$ \\
\hline 7 & The range of remote control via Blutooth & up to $100 \mathrm{~m}$ \\
\hline 8 & The range of remote control via $\mathrm{WiFi}$ & up to $300 \mathrm{~m}$ \\
\hline 9 & $\begin{array}{l}\text { The ultrasonic sensor (determination of distance to } \\
\text { objects) }\end{array}$ & up to $50 \mathrm{~m}$ \\
\hline 10 & $\begin{array}{l}\text { Voltage / capacity / continuous operation time of } \\
\text { battery R. O. }\end{array}$ & $\begin{array}{c}7-10 \mathrm{~B} \\
2 \times 3,7 \mathrm{~B} \times 4800 \mathrm{~A} / \mathrm{h} \\
\text { up to } 0.5 \mathrm{~h}\end{array}$ \\
\hline 11 & The range of video surveillance systems & up to $300 \mathrm{~m}$ \\
\hline 12 & $\begin{array}{l}\text { The ability to install various sensors (radiation, } \\
\text { temperature, humidity, illuminance, etc.) }\end{array}$ & Yes \\
\hline 13 & Indication of sensors on the robot & $16 * 2$ blue LCD screen \\
\hline 14 & Moisture & No \\
\hline 15 & The possibility of two-way voice communication & Yes \\
\hline 16 & Panoramic view of the area (rotation only) & $180^{0}$ \\
\hline 17 & Installed sensors for dangerous concentrations of gas & $\begin{array}{l}\text { MQ-2 Sensor to detect combustible gas and smoke } \\
\text { (propane, methane, n-butane), smoke (suspended } \\
\text { particles resulting from combustion) of hydrogen. } \\
\text { MQ-3 Sensor for vapour detection alcohol } \\
\text { C2H5OH } \\
\text { MQ-4 Sensor detect natural gas and methane } \\
\text { (CH4) in the air, vapors of alcohol, cigarette and } \\
\text { cooking smoke. } \\
\text { MQ-5 Sensor for detecting liquefied (LPG), natural } \\
\text { and coke oven gas. } \\
\text { Used for alarm gas leaks in the home and in the } \\
\text { workplace. } \\
\text { MQ-6 Sensor for detecting LPG, ISO-butane, } \\
\text { butane. } \\
\text { (natural gas, petroleum gas, butane, propane) } \\
\text { MQ-7 Analog sensor to detect carbon monoxide } \\
\text { (CO). } \\
\text { Used to detect CO2 at the plant, when carrying out } \\
\text { underground work in the laboratory and scientific } \\
\text { works. It can detect CO-gas concentrations in the } \\
\text { range of } 20 \text { to } 2000 \text { ppm. } \\
\text { MQ-8 Sensor for detecting Hydrogen (H2) gas and } \\
\text { coke oven gas. }\end{array}$ \\
\hline
\end{tabular}

ISPC The development of applied research, 


\begin{tabular}{l|lrl|l|ll} 
& ISRA (India) & $=\mathbf{1 . 3 4 4}$ & SIS (USA) & $=\mathbf{0 . 9 1 2}$ & ICV (Poland) & $=\mathbf{6 . 6 3 0}$ \\
Impact Factor: & ISI (Dubai, UAE) $=\mathbf{0 . 8 2 9}$ & PUHL (Russia) $=\mathbf{0 . 2 3 4}$ & PIF (India) & $=\mathbf{1 . 9 4 0}$ \\
& GIF (Australia) & $\mathbf{0 . 5 6 4}$ & ESJI (KZ) & $=\mathbf{1 . 0 4 2}$ & IBI (India) & $\mathbf{= 4 . 2 6 0}$ \\
& JIF & $\mathbf{1 . 5 0 0}$ & SJIF (Morocco) & $=\mathbf{2 . 0 3 1}$ & & \\
\hline
\end{tabular}

\begin{tabular}{|l|l|}
\hline $\begin{array}{l}\mid \begin{array}{l}\text { MQ-9 Combined analog gas sensor (CO+CO or } \\
\text { whether it is in the air. } \\
\text { CNG+LPG) } \\
\text { (propane, methane, n-butane) and carbon monoxide } \\
\text { (CO). }\end{array} \\
\text { MQ135 gas Detector } \\
\text { In addition to carbon dioxide, the sensor also reacts } \\
\text { to the presence of other gases: carbon monoxide, } \\
\text { ammonia, benzene, nitrogen oxides and vapors of } \\
\text { alcohol. Apply for permanent air quality monitoring } \\
\text { in industrial or domestic premises. }\end{array}$ \\
\hline
\end{tabular}

\section{Materials and Methods}

Programming of the microcontroller the robot will implement the designed program:

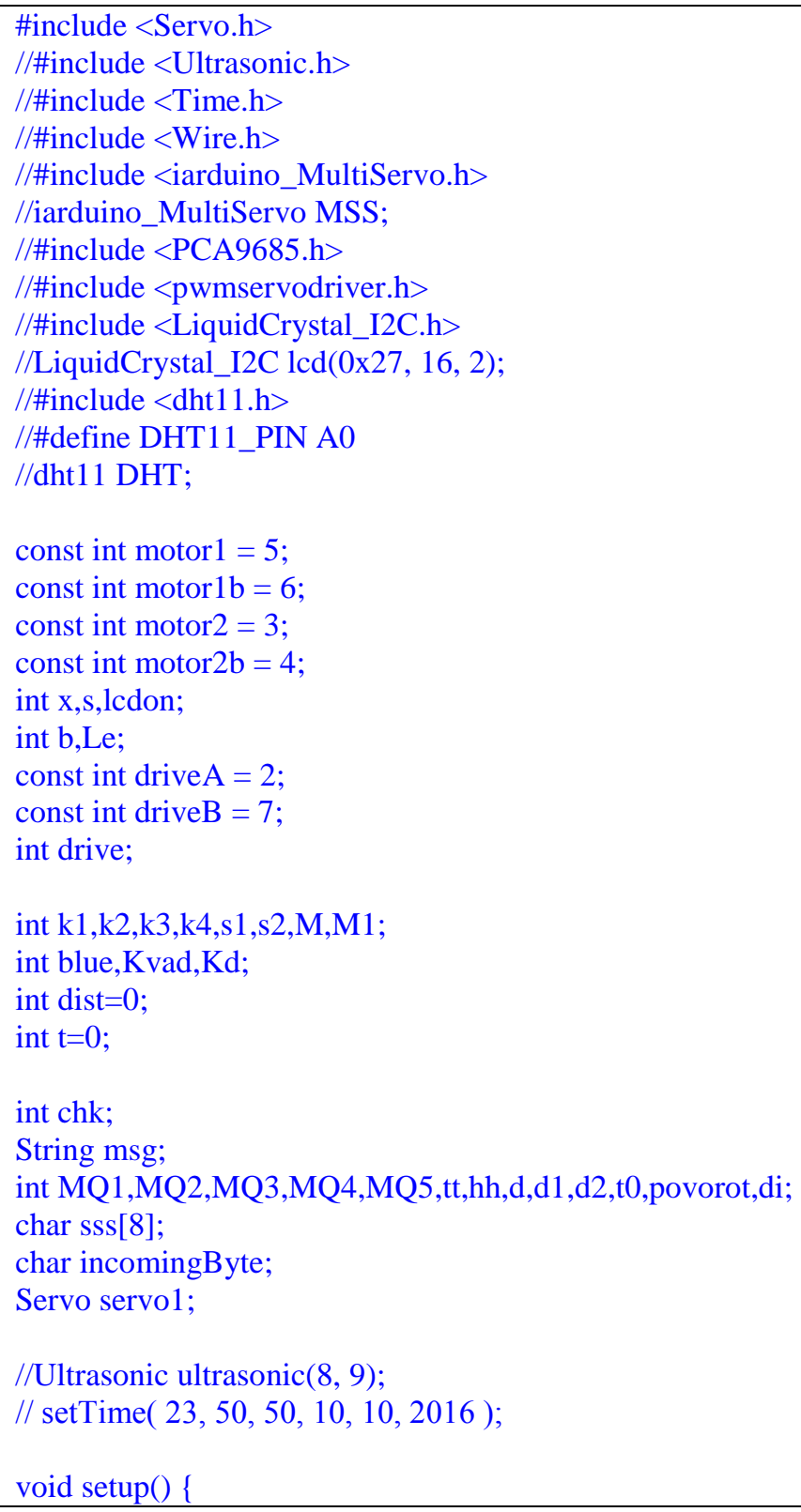




\begin{tabular}{|c|c|c|c|c|c|c|}
\hline Impact Factor: & $\begin{array}{l}\text { ISRA (India) } \\
\text { ISI (Dubai, UAE } \\
\text { GIF (Australia) } \\
\text { JIF }\end{array}$ & $\begin{array}{l}=1.344 \\
=0.829 \\
=0.564 \\
=1.500\end{array}$ & $\begin{array}{l}\text { SIS (USA) } \\
\text { PИНЦ (Russia) } \\
\text { ESJI (KZ) } \\
\text { SJIF (Morocco) }\end{array}$ & $\begin{array}{l}=0.912 \\
=0.234 \\
=1.042 \\
=2.031\end{array}$ & $\begin{array}{l}\text { ICV (Poland) } \\
\text { PIF (India) } \\
\text { IBI (India) }\end{array}$ & $\begin{array}{l}=6.630 \\
=1.940 \\
=4.260\end{array}$ \\
\hline
\end{tabular}

// put your setup code here, to run once:

servo1.attach(11);

Serial.begin(9600);

pinMode(motor1, OUTPUT);

pinMode(motor1b, OUTPUT);

pinMode(motor2, OUTPUT);

pinMode(motor2b, OUTPUT);

pinMode(driveA, OUTPUT);

pinMode(driveB, OUTPUT);

pinMode(10, OUTPUT);

//pinMode(A0, INPUT);

pinMode(A1, INPUT);

pinMode(A2, INPUT);

pinMode(A3, INPUT);

pinMode(A4, INPUT);

pinMode(A5, INPUT);

$\mathrm{x}=0 ;$ drive $=0 ;$

blue $=1$;

digitalWrite(10, HIGH);

$\mathrm{s}=66$;

$\mathrm{s} 1=75$;

s2 $=130$;

servo1.write(88);//60-130

Le $=0$;

$\mathrm{di}=0$;

$\mathrm{Kvad}=0$;

lcdon=0;

$\mathrm{M} 1=0$;

$\mathrm{t}=0$;

drive $=0$;

\}

void loop() \{ // put your main code here, to run repeatedly:

if $(\mathrm{Le}==1)$

MQ1 = analogRead(A1);

MQ2 = analogRead(A2);

MQ3 = analogRead(A3);

MQ4 = analogRead(A4);

MQ5 = analogRead(A5);

$\mathrm{msg}$

"M"+String(MQ1)+"

+"R"+String(MQ5)+"

"+"N"+String(MQ2)+" "+"O"+String(MQ3)+" "+"P"+String(MQ4)+"

Serial.print(msg);

$\mathrm{t}=\mathrm{t}+1$;

if $(\mathrm{t}>50)\{\mathrm{t}=0 ; \mathrm{Le}=0 ;$ digitalWrite $(10, \mathrm{HIGH}) ;\}$

if (Serial.available ()$>0)\{$

incomingByte $=$ Serial.read () ;

if(incomingByte $==$ 'U') $\{\mathrm{Le}=1$; digitalWrite $(10, \mathrm{LOW}) ;\}$

if (incomingByte $==$ 'u' $)\{$ Le=0; digitalWrite $(10, \mathrm{HIGH}) ;\}$

//if(incomingByte == 'X')\{lcdon=1; lcd.backlight ()$;\}$

ISPC The development of applied research, 
//if(incomingByte == 'x') $\{$ lcdon=0; lcd.noBacklight(); $\}$

$/ /$ if(incomingByte $\left.=={ }^{\prime} \mathrm{V}^{\prime}\right)\{\mathrm{b}=1 ;\}$

$/ /$ if(incomingByte $==$ ' $^{\prime}$ ') $\{\mathrm{b}=0 ;\}$

$/ /$ if $\left(\right.$ incomingByte $\left.=={ }^{\prime} \mathrm{V}^{\prime}\right)\{\mathrm{di}=1 ;\}$

$/ /$ if (incomingByte $==$ 'u' $\left.^{\prime}\right)\{\mathrm{di}=0$; $\}$

if(incomingByte $\quad==\quad 2$ ') $\quad\{\quad$ servo1.write(140); $\}$ if(incomingByte $==\quad 3$ ') \{ servo1.write(120); \}if(incomingByte $==\quad 4$ ') $\quad\{$ servo1.write $(100)$; $\}$ if $\left(\right.$ incomingByte $\left.==\quad 5^{\prime}\right) \quad\{$ servo1.write(80);

if(incomingByte == '6') \{ servo1.write(66); $\}$ if(incomingByte == '7') \{ servo1.write(40); $\}$ if(incomingByte $=={ }^{\prime} 8$ ') $\{$ servo1. write(20); $\}$ if(incomingByte $==$ '9') $\{$ servo1.write $(0) ;\}$

//if(incomingByte $==\quad$ '2') $\quad\{\quad$ MSS.servoWrite(15,140); $\}$ if(incomingByte $==\quad$ '3') \{ MSS.servoWrite $(15,120)$; $\}$ if(incomingByte == '4') $\{$ MSS.servoWrite $(15,100)$; $\}$ if(incomingByte $==$ '5') \{ MSS.servoWrite $(15,80) ;\}$

//if(incomingByte $\left.==\quad 6^{\prime}\right) \quad\{\quad$ MSS.servoWrite $(15,66) ;\}$ if(incomingByte $\left.==\quad 7^{\prime}\right) \quad\{$ MSS.servoWrite $(15,40)$; $\}$ if(incomingByte $==$ ' $^{\prime}$ ') $\{$ MSS.servoWrite $(15,20)$; $\}$ if(incomingByte $==$ '9') \{ MSS.servoWrite(15,0); $\}$

if(incomingByte $==$ 'F' $\{$ drive $=1 ; \mathrm{M}=1$; motorClear ()$; \operatorname{motor}() ; \quad\} / /$ вперед

if(incomingByte $==$ 'B' $)\{$ drive $=1 ; \mathrm{M}=2 ; \operatorname{motorClear}() ; \operatorname{motorB}() ; \quad\} / /$ назад

if $\left(\right.$ incomingByte $==$ 'L'$^{\prime}$ \{drive $=1 ; \mathrm{M}=3$; motorClear ()$\left.; \operatorname{motorL}() ;\right\}$

if (incomingByte $==$ 'R' $^{\prime}$ \{drive $=1 ; \mathrm{M}=4$; motorClear ()$\left.; \operatorname{motorR}() ;\right\}$

if $($ drive $==1)\{\operatorname{motorOn}() ; \operatorname{delay}(20) ; \operatorname{motorOff}() ;\}$;

if $(\mathrm{di}==1)\{$

while (incomingByte > 'B' || incomingByte < 'B' ) \{

if (Serial.available ()$>0)\{$ //если пришли данные

incomingByte $=$ Serial.read ()$; / /$ считываем байт

delay(30); \};

//if (ultrasonic.Ranging $(\mathrm{CM})<8 \& \&$ ultrasonic. Ranging $(\mathrm{CM})>0)\{$ incomingByte $=$ 'u'; $\}$;

\}

motorClear();

motorOff();

\}

\} else $\{$ drive $=0 ;\}$

motorClear();

\}

void motorOnR()\{

digitalWrite(driveA, LOW);

digitalWrite(driveB, HIGH);

\}

void motorOnL() \{

digitalWrite(driveA, HIGH);

digitalWrite(driveB, LOW);

\}

void motorOn()\{

digitalWrite(driveA, HIGH);

digitalWrite(driveB, $\mathrm{HIGH}$ );

\}

void motorOff() \{

digitalWrite(driveA, LOW); 


\begin{tabular}{|c|c|c|c|c|c|c|}
\hline Impact Factor: & $\begin{array}{l}\text { ISRA (India) } \\
\text { ISI (Dubai, UAE } \\
\text { GIF (Australia) } \\
\text { JIF }\end{array}$ & $\begin{array}{l}=1.344 \\
=0.829 \\
=0.564 \\
=1.500\end{array}$ & $\begin{array}{l}\text { SIS (USA) } \\
\text { PИНЦ (Russia) } \\
\text { ESJI (KZ) } \\
\text { SJIF (Morocco) }\end{array}$ & $\begin{array}{l}=0.912 \\
=0.234 \\
=1.042 \\
=2.031\end{array}$ & $\begin{array}{l}\text { ICV (Poland) } \\
\text { PIF (India) } \\
\text { IBI (India) }\end{array}$ & $\begin{array}{l}=6.630 \\
=1.940 \\
=4.260\end{array}$ \\
\hline
\end{tabular}

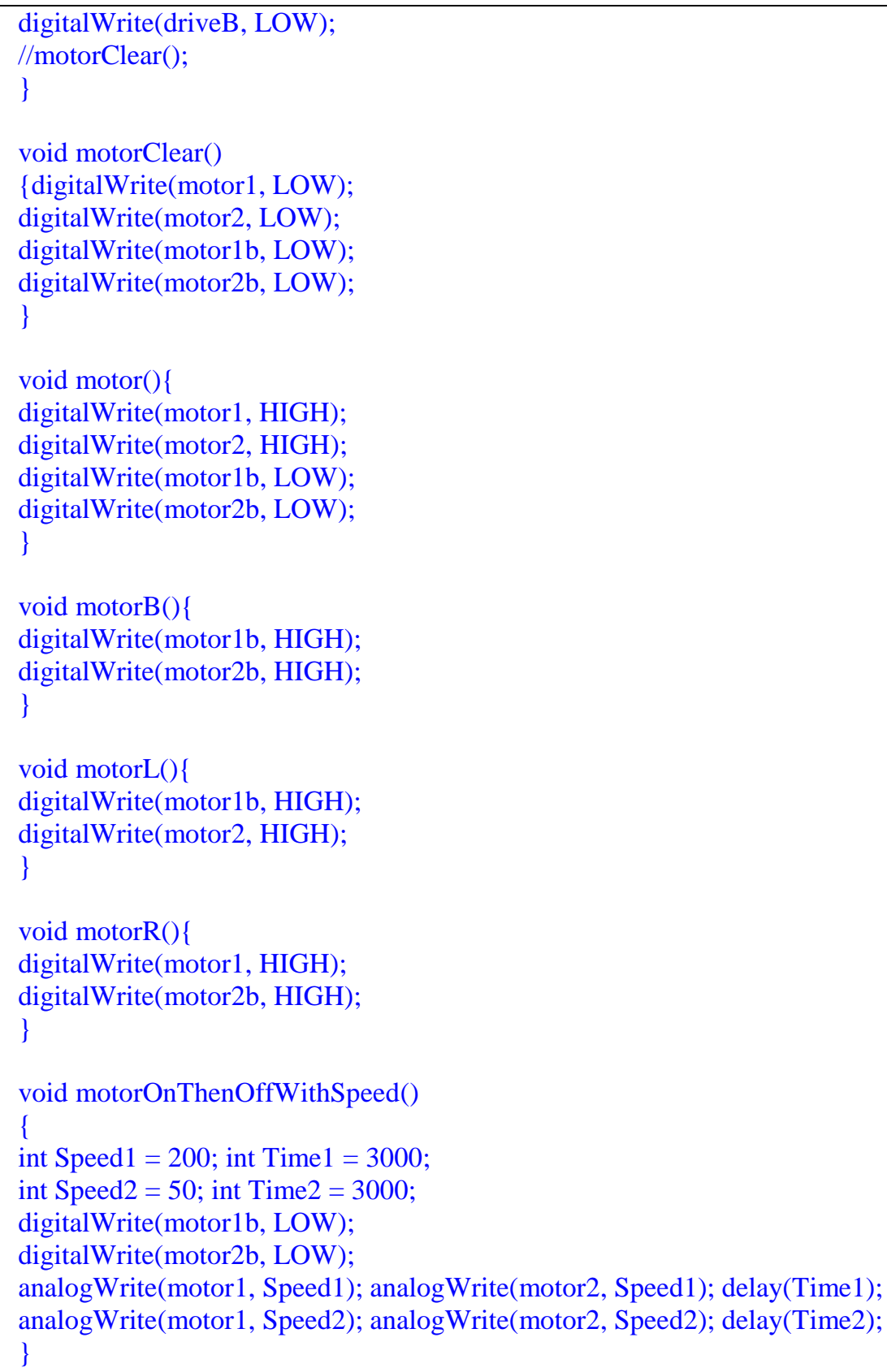




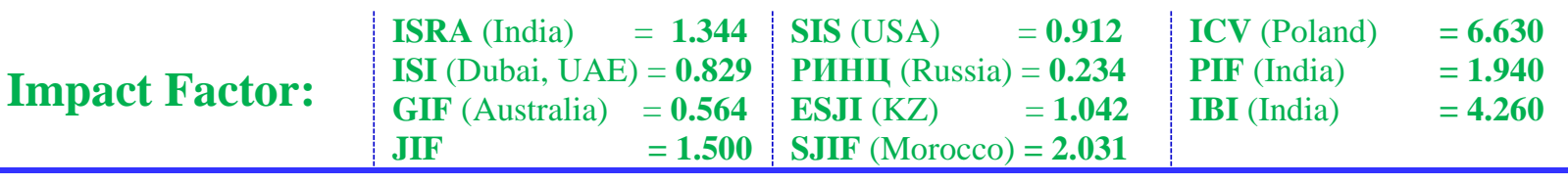

\section{Перо S Pen извлечено}
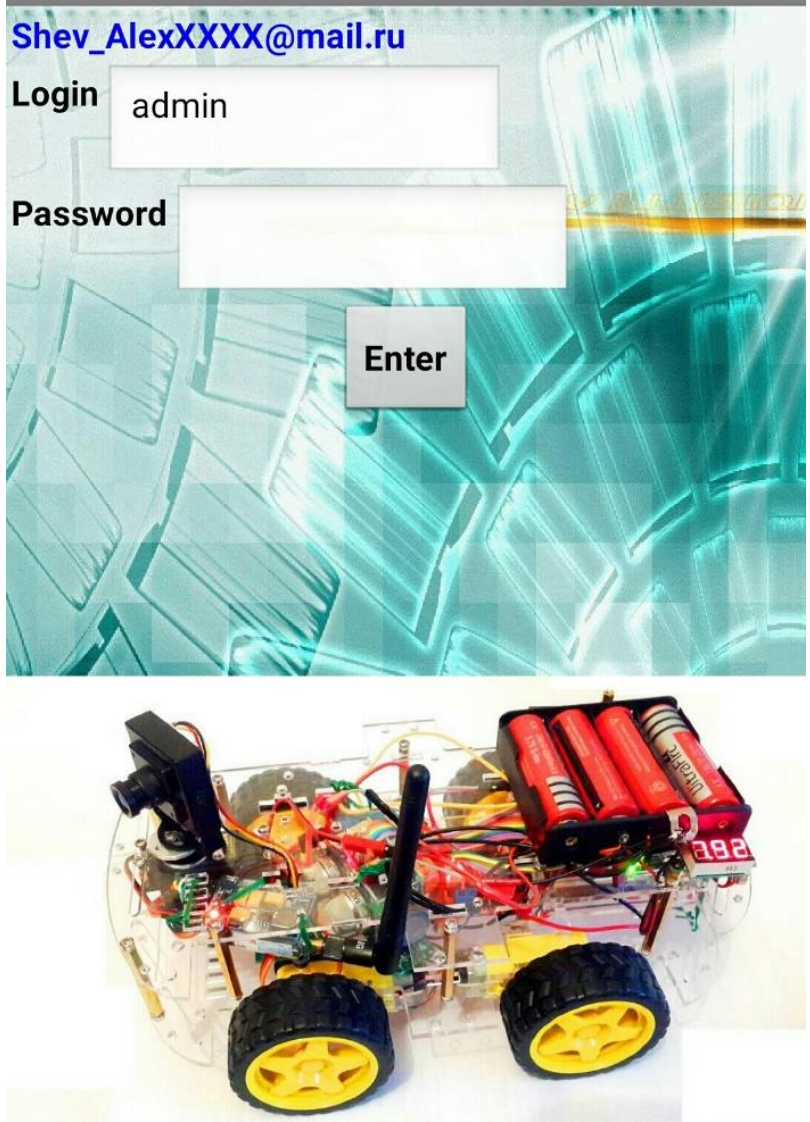

\section{D@ \&}

\section{约行 $480 \%$ I:16 PM}

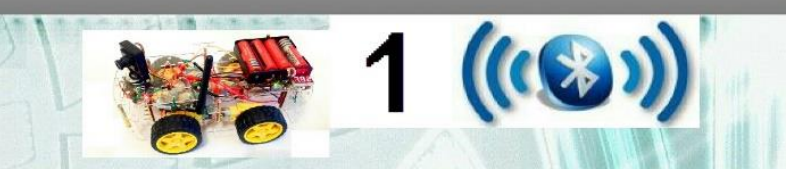

No device

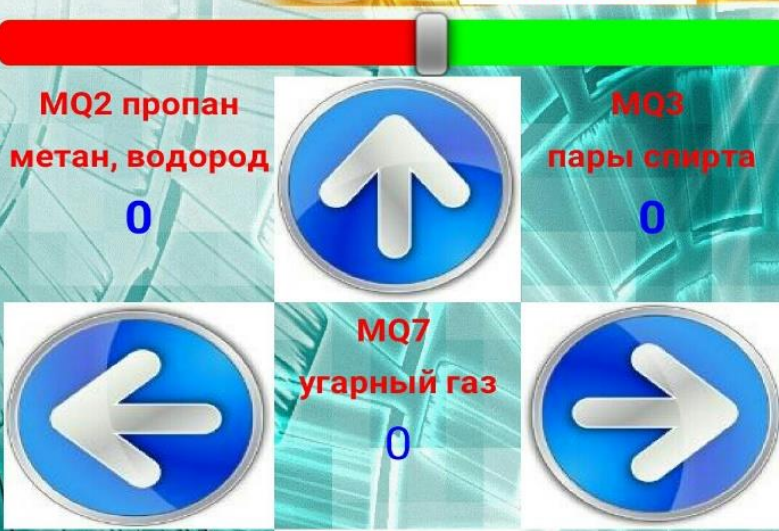

MQ135 аммиак

бензол, $\mathrm{CO} 2$

0

Figure 2 - Developed control program.

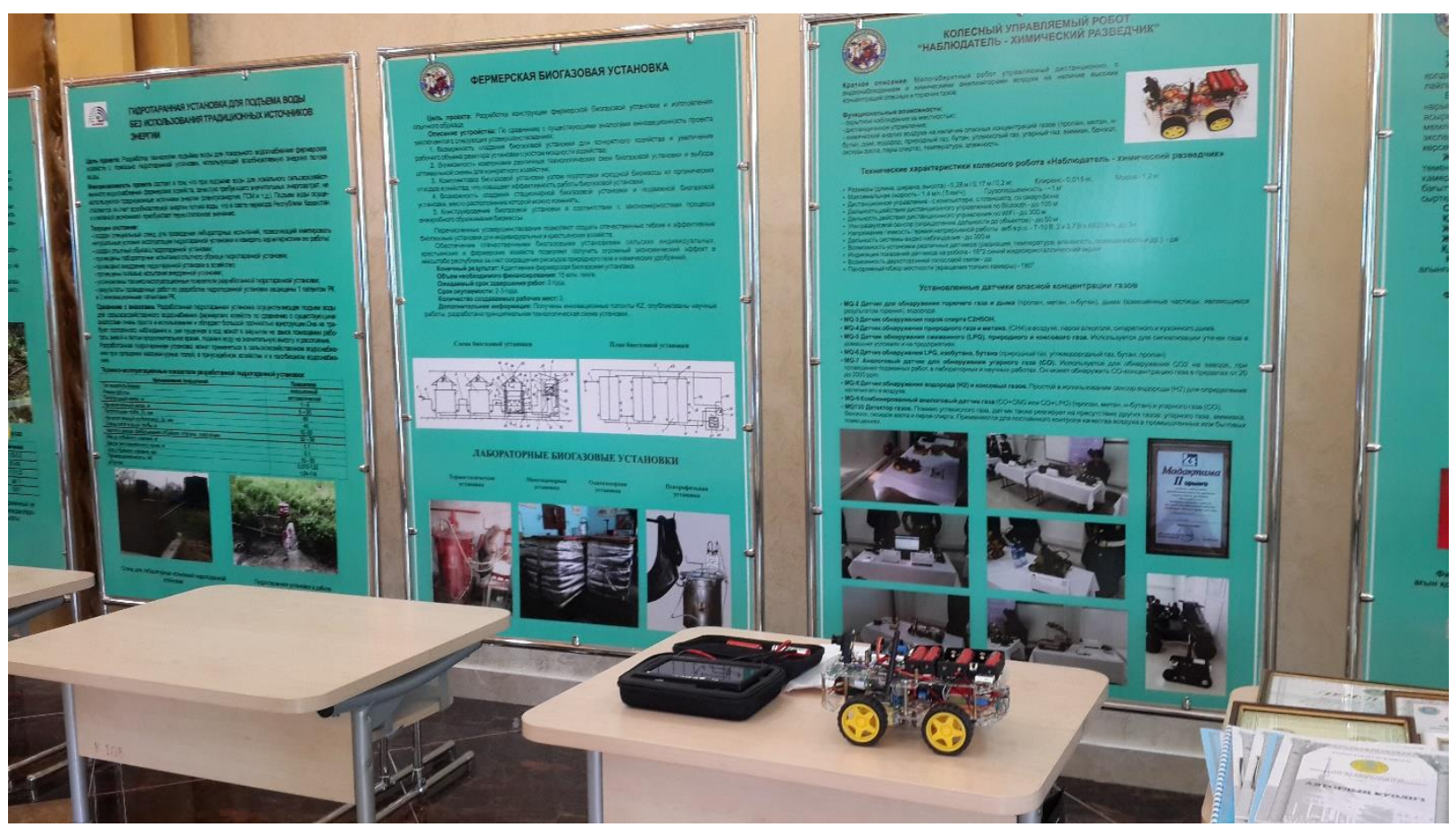

Figure 3 - Stand and demonstration of the robot (August 23, 2016) .

ISPC The development of applied research, Harrisburg, USA 


\begin{tabular}{l|lrl|l|ll} 
& ISRA (India) & $=\mathbf{1 . 3 4 4}$ & SIS (USA) & $=\mathbf{0 . 9 1 2}$ & ICV (Poland) & $=\mathbf{6 . 6 3 0}$ \\
Impact Factor: & ISI (Dubai, UAE) $=\mathbf{0 . 8 2 9}$ & PUHL (Russia) $=\mathbf{0 . 2 3 4}$ & PIF (India) & $=\mathbf{1 . 9 4 0}$ \\
& GIF (Australia) & $\mathbf{0 . 5 6 4}$ & ESJI (KZ) & $=\mathbf{1 . 0 4 2}$ & IBI (India) & $=\mathbf{4 . 2 6 0}$ \\
& JIF & $\mathbf{1 . 5 0 0}$ & SJIF (Morocco) & $=\mathbf{2 . 0 3 1}$ & & \\
\hline
\end{tabular}

\section{Conclusion}

This project to develop a gas analyzer station based on the robot participated in the competition of robotics in the framework of military training Aibyn 2016 and took 2nd place, as well as in the exhibition of achievements of 23 August 2016.
The developed robot is able to reach inaccessible places to the operator transmitting video as well as producing chemical reconnaissance of combustible gases.

\section{References:}

1. (2016) Collect the robots creep on the Arduino - "Hacker" Available: xakep.ru"2014/10/30/robots-arduino/ (Accessed: 10.08.2016).

2. (2016) Ready Arduino robots robotics Entertaining Available: edurobots.ru"the Finished Arduino robots (Accessed: 10.08.2016).

3. (2016) Arduino. Finished project "Robotmachine RoboCar4W" Available: tim4dev.com"arduino-instruction-projectrobocar4w/ (Accessed: 10.08.2016).

4. (2016) Arduino robot... Hardware platform Arduino Forum Available: arduino.ru"Forums"Arduino robot for beginners (Accessed: 10.08.2016).

5. (2016) Meet our mini robot for arduino... Available: arduinokit.ru"arduino/arduino...our...robot... arduino... (Accessed: 10.08.2016).

6. (2016) RT0008 Robot on Arduino Uno Available: mysku.ru"All records"China Stores"36376.html (Accessed: 10.08.2016).

7. (2016)Collect simple robot for Arduino Available: uSamodelkina.ru"6754...robotana-arduino.html (Accessed: 10.08.2016).
8. (2016) Arduino robot with his own hands Available: shagir.ru"robot-arduino-selvesrukami (Accessed: 10.08.2016).

9. (2016) Robot On Arduino with Your Hands the World of robotics Available: informatikm.ru"...robototehniki/robot-na-arduino... (Accessed: 10.08.2016).

10. (2016) Step-by-step instructions for creating robots - Robotics Available: robotics.ua"build_robot/medium_robots (Accessed: 10.08.2016).

11. (2016) SERVODROID Robots, robotics with their hands... Available: servodroid.ru"index/programmirovanie_ard uino/0-87 (Accessed: 10.08.2016).

12. (2016) Arduino / Search by tags / RoboCraft. Robots? It's easy! Available: robocraft.ru"tag/Arduino (Accessed: 10.08.2016).

13. (2016) Robot vacuum cleaner on Arduino / Geektimes Available: geektimes.ru Robot vacuum cleaner on Arduino (Accessed: 10.08.2016).

14. (2016) Robot Arduino - Brazed Available: payaem.ru"robot-na-arduino.html (Accessed: 10.08.2016). 